\title{
涉及碳一氟键断裂与重组的氟化重排反应：由炔丙基氟制备官能团化 偕二氟烯丙基化合物
}

\author{
倪传法 胡金波*
}

(中国科学院上海有机化学研究所 上海 200032)

\section{Fluorinative Rearrangement Involving Cleavage and Recombination of Carbon-Fluorine Bond: Synthesis of Functionalized Allylic gem-Difluorides from Propargylic Fluorides}

\author{
$\mathrm{Ni}$, Chuanfa $\mathrm{Hu}$, Jinbo* \\ (Shanghai Institute of Organic Chemistry, Chinese Academy of Sciences, Shanghai 200032)
}

有机氟化学中有一个有趣的现象: 当多氟烷基碳上 的碳-氟键发生断裂与重组时, 氟原子倾向于从少氟的 碳迁移到多氟的碳, 从而使得氟原子聚集在同一个碳原 子上 ${ }^{[1]}$. 例如, $\mathrm{CF}_{2} \mathrm{ClCFCl}_{2}$ 在路易斯酸 $\mathrm{AlBr}_{3}$ 的作用下, 高选择性地生成热力学更稳定的产物 $\mathrm{CF}_{3} \mathrm{CCl}_{3}$ (Scheme 1a $)^{[1]}$. 这一反应发生的原因是: $\mathrm{sp}^{3}$-碳上的氟原子取代 越多, “负” 超共轭作用(“negative” hyperconjugation)越 强, 使得体系能量更低(Scheme $1 \mathrm{~b})^{[1]}$. 但是在有机合成 中, 分子内发生碳-氟键断裂与重组的反应并不常见 ${ }^{[2]}$, 尤其是重组后形成多氟代碳中心的反应更为少见.

(a)

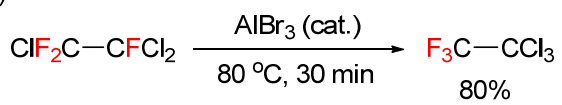

(b)

$$
\mathrm{F}_{\mathrm{F}} \longleftrightarrow \mathrm{F}^{-} \mathrm{F}_{\mathrm{F}}^{+} \longleftrightarrow \mathrm{F}_{\mathrm{F}^{+}}
$$

图式 1 (a)路易斯酸诱导的氟迁移反应和(b) “负” 超共轭作 用对二氟亚甲基的稳定化作用

Scheme 1 (a) Lewis acid-induced fluorine migration, and (b) stabilization of difluoromethylene group by "negative" hyperconjugation

经典的 Meyer-Schuster 重排反应指的是炔丙醇在酸 催化作用下, 经过表观 1,3-羟基迁移, 重排为联烯醇, 并互变异构为烯酮转化 ${ }^{[3]}$. 该人名反应有多个变体, 其 中一个变体是向炔丙醇(酯)反应体系中加入亲电试剂,
通过捕获联烯醇(酯)中间体或者先对碳碳参键亲电加成 再发生表观 1,3-氧迁移重排, 从而得到各种 $\alpha$-官能团化 烯酮(Scheme 2a) $)^{[4-6]}$. 受这一反应模式的启发, 中山大 学化学学院赵晓丹课题组近期报道了一例类似于 Meyer-Schuster 重排反应的氟化反应 ${ }^{[7]}$, 很好地展示了 分子内碳-氟键断裂与重组反应在含氟复杂分子合成中 的实用性(Scheme $2 b$ ).

(a)

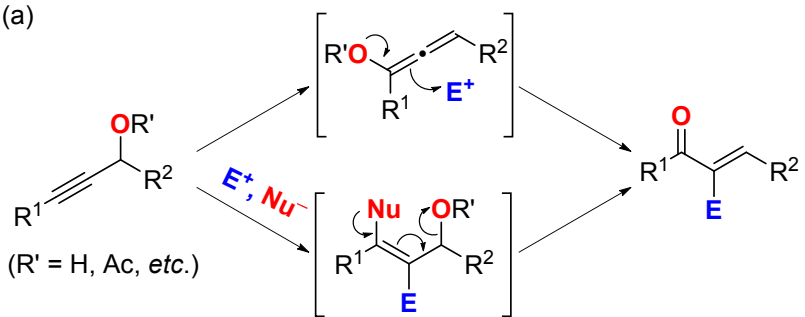

(b)

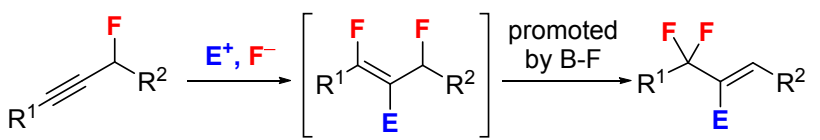

图式 2 (a)亲电试剂作用下的 Meyer-Schuster 重排反应和(b) 与 Meyer-Schuster 重排反应类似的氟化重排反应

Scheme 2 (a) Meyer-Schuster rearrangement under the action of electrophiles, and (b) fluorinative Meyer-Schuster-Like rearrangement

氟化重排反应从炔丙基氟 ${ }^{[8]}$ 出发, 以四氟硼酸-乙 醚作为氟源兼碳一氟键活化试剂，在二芳基硫醚对酰亚 胺类亲电试剂的催化活化下，通过对碳一碳参键的官能

* Corresponding author. E-mail: jinbohu@sioc.ac.cn. Published online August 3, 2020. 
才化-氟化生成 1,3-二氟烯丙基化合物，继而发生三氟 化喼活化烯丙位碳一氟键进行的表观 1,3-氟迁移重排, 最终得到含有二氟亚甲基的多取代 1,1-二氟烯丙基化合 物(Scheme 3) ${ }^{[7]}$. 作者不但分离到了 1,3-二氟-2-碘代烯 丙基化合物, 验证了其重排反应性的可行性, 还通过空 白实验排除了炔丙基氟首先异构化为联烯基氟的可能 性.

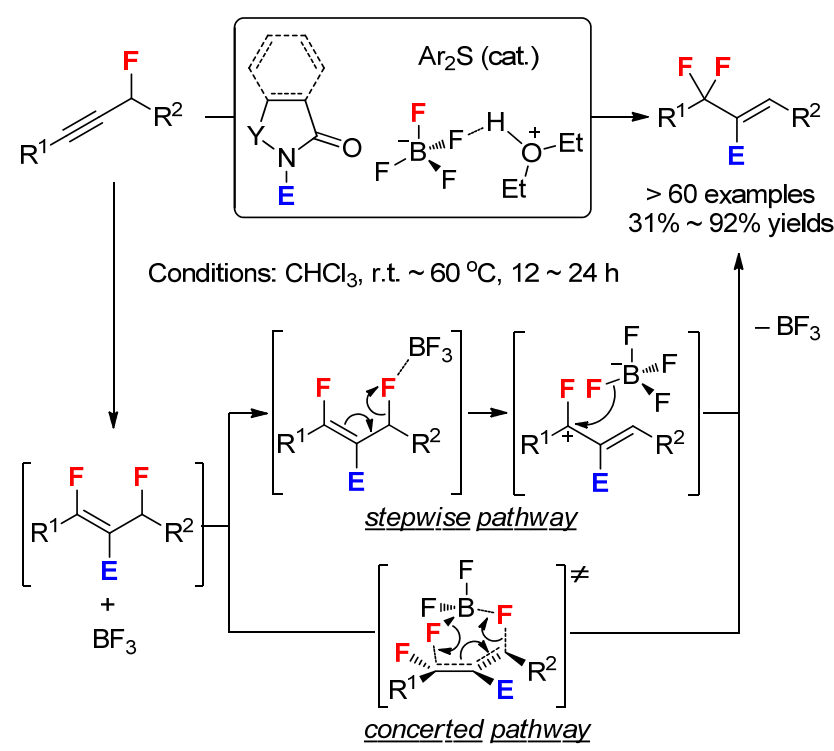

图式 3 炔丙基氟发生氟化重排的反应条件以及发生表观 1,3氟迁移的可能路径

Scheme 3 Reaction conditions for fluorinative rearrangement of propargylic fluorides and possible pathways for formal 1,3-fluorine migration

上述反应适用于一级和二级炔丙基氟的氟化重排, 具有比较广泛的底物范围以及以单一的区域选择性, 所 引入的官能团除了碘, 还包括溴以及三氟甲硫基(图 1) ${ }^{[7]}$ 当二级炔丙基氟作为底物时, 反应还具有优秀的顺式立 体选择性. 很显然, 亲电试剂对碳碳参键加成这一步的 高区域选择性以及重排这一步的高立体选择性均得益 于氟原子取代基的影响.

值得指出的是, 偕二氟烯丙基单元不但是一些药物 和生物活性分子中的结构片段, 而且可以发生各种转化 用于合成结构多样的含氟分子 ${ }^{[7]}$. 目前, 偕二氟烯丙基 化合物的合成方法非常有限, 主要包括偕二氟亚甲基化
合物参与的交叉偶联以及 $\alpha, \beta$-烯酮的脱氧氟化 ${ }^{[7]}$. 因此, 作者通过烯基碘参与的偶联反应，进一步展示了用他们 发展的策略所合成的 1,1-二氟-2 碘代烯丙基化合物的广 泛合成用途 ${ }^{[7]}$.
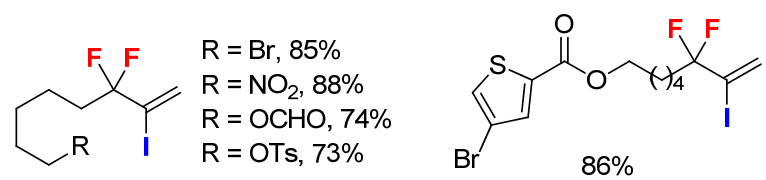<smiles>C=C(I)C(F)(F)c1cc(Br)cc(C(C)=O)c1</smiles><smiles>C=C(I)C(F)(F)c1ccc(C(O)(C(F)(F)F)C(F)(F)F)cc1</smiles>

$70 \%$

$50 \%$<smiles>C/C=C(\F)C(F)(F)c1ccccc1</smiles>

$E=I, 75 \%$

$\mathrm{E}=\mathrm{Br}, 61 \%$<smiles>C=C(SC(F)(F)F)C(F)(F)[Y10]COC(=O)/C=C/c1ccccc1</smiles>

$89 \%$

图 1 从炔丙基氟出发合成的一些代表性偕二氟烯丙基化合 物

Figure 1 Some representative allylic gem-difluorides prepared from propargylic fluorides

总之, 赵晓丹课题组的上述研究工作不但丰富了偕 二氟烯丙基化合物的合成方法，而且为发展二氟亚甲基 化合物的合成方法研究提供了新思路. 我们期望在将来 能够发展出直接从炔丙醇出发的 Meyer-Schuster 类型的 脱氧氟化反应.

\section{References}

[1] Kirsch, P. Modern Fluoroorganic Chemistry: Synthesis, Reactivity Applications, 2nd ed., Wiley-VCH, Weinheim, 2013, p. 41, p. 58.

[2] For examples of fluorine-migration reaction: (a) Luo, T.; Zhang, R.; Zhang, W.; Shen, X.; Umemoto, T.; Hu, J. Org. Lett. 2014, 16, 888. (b) Luo, T.; Zhang, R.; Shen, X.; Zhang, W.; Ni, C.; Hu, J. Dalton Trans. 2015, 44, 19636.

[3] Swaminathan, S.; Narayanan, K. V. Chem. Rev. 1971, 71, 429

[4] Engel, D. A.; Dudley, G. B. Org. Biomol. Chem. 2009, 7, 4149.

[5] Zhu, Y.; Sun, L.; Lu, P.; Wang, Y. ACS Catal. 2014, 4, 1911.

[6] Roy, D.; Tharra, P.; Baire, B. Asian J. Org. Chem. 2018, 7, 1015.

[7] Liao, L.; An, R.; Li, H.; Xu, Y.; Wu, J.-J.; Zhao, X. Angew. Chem., Int. Ed. 2020, 59, 11010.

[8] Pacheco, M. C.; Purser, S.; Gouverneur, V. Chem. Rev. 2008, 108, 1943.

(Li, L.) 